\title{
The Volume Operator in Loop Quantum Gravity
}

\author{
Johannes Brunnemann*t \\ XRG Simulation GmbH, Harburger Schlossstraße 6-12, 21079 Hamburg, Germany \\ E-mail: brunnemann@xrg-simulation.de
}

The volume operator is a central object in loop quantum gravity (LQG). It is part of all matter Hamiltonians as well as the Hamilton constraint encoding the dynamics of the theory. Therefore it is mandatory to understand its spectral properties. This lecture gives an introduction to basic techniques necessary in order to perform such an analysis. Moreover results on the volume operator are presented which point towards a better understanding of a presumably combinatorial footing of LQG.

The plan of the lecture is as follows: After a short introduction, we outline the implementation of the classical volume functional of a region in 3-space as an operator on the kinematic Hilbert space of LQG. Then we introduce techniques crucial for computing the matrix elements of the volume operator: First, aspects of the representation theory of $S U(2)$ are explained which make it possible to use techniques from the recoupling theory of angular momenta. Second, the mathematical concept of an oriented matroid is introduced and used in order to describe the dependence of the matrix elements on non-diffeomorphic properties at vertices of embedded graphs. The according effect on spectral properties is shown. In the last section a short summary is given combined with an outlook on future research. A short selection of literature is presented a the end of these notes, which is intended as a starting point for reading.

We would like to stress, that the focus of these lectures is to give basic ideas and concepts to the reader, it cannot replace a complete, mathematically rigorous introduction [1]. However we aim at giving the reader an initial competence for starting his or her own studies on the important topic of quantized volume and its fascinating conceptual consequences.

3rd Quantum Gravity and Quantum Geometry School,

February 28 - March 13, 2011

Zakopane, Poland

\footnotetext{
*Speaker.

${ }^{\dagger}$ Former affiliation: Paderborn University, Mathematics Dept. E-mail: johannes.brunnemann@math.upb.de
} 


\section{Motivation - Why Quantized Volume?}

The classical starting point for the construction of loop quantum gravity (LQG) is the initial value formulation of general relativity. Four dimensional spacetime $\mathfrak{M}$ is foliated into three dimensional spatial Cauchy hypersurfaces $\Sigma_{t}$ parametrized by the time parameter $t \in \mathbb{R}$. Then, on each $\Sigma_{t}$ it can be shown that the validity of Einstein's equations on $\mathfrak{M}$ is equivalent to imposing three so called spatial diffeomorphism constraints $C_{a}(x)$ (here $a, \ldots, d=1,2,3$ denote spatial coordinates, $i, \ldots, n=1,2,3$ denote coordinates on the Lie algebra $s u(2))$ and the Hamilton constraint $C(x)$ at each $x \in \Sigma_{t}$. The formulation of the theory in terms of Ashtekar variables furthermore introduces the Gauss constraints $\mathscr{G}_{i}(x)$. According to this procedure the 3-metric $q$ and the extrinsic curvature $K$ on $\Sigma_{t}$ are induced from the 4-metric $g$ on $\mathfrak{M}$. In order to construct a quantum theory of gravity, classical differential geometric objects have to be turned into operators. For this, the quantum version of the volume functional $V_{R}$ of a region $R \subseteq \Sigma_{t}$

$$
V_{R}=\int_{R} \sqrt{\operatorname{det} q}(x) d^{3} x
$$

is a central object: It associates a volume to spatial regions and occurs in all matter Hamiltonians [1]. Even more, the Hamilton constraint encoding the dynamics of LQG crucially depends on it. In de-parametrized models the volume expression itself becomes part of physical quantities [10].

In this lecture, I will only discuss the original approach to the volume operator as proposed by $[4,5,6]$. However due to its importance there are also alternative approaches: see the lectures on spinfoams and loop quantum cosmology as well as for example [12, 13, 11].

\section{Regularization and Implementation on the Kinematic Hilbert Space $\mathscr{H}_{0}$}

Choosing a coordinate chart on $\Sigma_{t}$, one gets for every $x \in \Sigma_{t}$ the components $q_{a b}(x)=e_{a}^{i}(x) e_{b}^{j}(x) \delta_{i j}$ of the 3-metric and $K_{a b}(x)=K_{a}^{i}(x) e_{b}^{j}(x) \delta_{i j}$ of the extrinsic curvature. Here $e_{a}^{i}(x)$ is a local triad, $K_{a}^{i}(x)=\beta^{-1}\left(A_{a}^{i}(x)-\Gamma_{a}^{i}(x)\right)$ and $\delta_{i j}$ is the Cartan-Killing metric on $s u(2)$. In the latter expression $\beta$ is the Barbero Immirzi parameter, which is chosen to be arbitrary real, but non zero. We will set it to 1 for convenience here and use Einstein's sum convention. Also we will drop the $\mathrm{x}$ - and t-dependence. $A_{a}^{i}$ is the Ashtekar connection, $\Gamma_{a}^{i}$ the spin connection resulting from the 3-metric $q$. Introducing densitized triads $E_{i}^{a}=\sqrt{\operatorname{det} q} e_{i}^{a}\left(e_{i}^{a}\right.$ denotes the inverse triad) (1.1) can be rewritten as

$$
V_{R}=\int_{R} d^{3} x \sqrt{\frac{1}{3 !}\left|\varepsilon_{a b c} \varepsilon^{i j k} E_{i}^{a}(x) E_{j}^{b}(x) E_{k}^{c}(x)\right|}=: \int_{R} d^{3} x \sqrt{|q(x)|},
$$

where $\varepsilon^{i j k}, \varepsilon_{a b c}$ are the total antisymmetric tensors $\left(\varepsilon^{123}=1, \varepsilon^{213}=-1\right.$ etc.). We have already seen in the the introductory lectures on LQG, how the densitized triads $E_{i}^{a}$ can be regularized and turned into flux operators $\widehat{E}_{S}^{i}$ as illustrated in figure 1 .

A similar regularization procedure can be applied in order to promote (2.1) to an operator acting on a spin network function $T_{\gamma}(A)$ ( $\gamma$ a graph embedded into $\Sigma, A \in \overline{\mathscr{A}}$ a generalized connection) contained in the kinematic Hilbert space $\mathscr{H}_{0}$. Starting point for the regularization is a choice of coordinate charts covering $\Sigma$. Then one introduces a cell decomposition of $\Sigma$ into cubes $c_{I}^{(L)}$ of 
coordinate edge length $L$, such that inside each cube there is at most one vertex $v$ of $\gamma$. Then the integral $\int_{R}$ decomposes into a sum over cube integrals $\sum_{I} \int_{c_{I}(L)}$. At each vertex $v$ we can w.l.o.g. choose all edges to be outgoing from $v$, because reorientation of edges corresponds to a unitary transformation on $\mathscr{H}_{0}$, which leaves the spectrum of any operator invariant. In the center of cubes $c_{I}^{(L)}$ containing a vertex $v$ we have for every edge triple $e_{I}, e_{J}, e_{K}$ intersecting at $v$ the configuration shown in figure 2 .

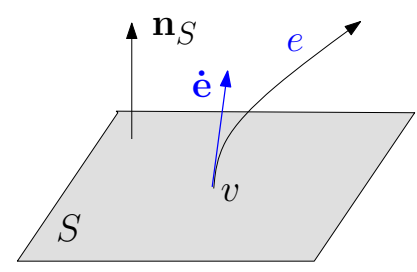

Figure 1: Action of $E_{S}^{i}=\int_{S} d^{2} s n_{s}^{a}(s) E_{a}^{i}(s)$ on a single edge spin network function $f_{e}$ : $\widehat{E}_{S}^{i} f_{e}=\varepsilon\left(\dot{\mathbf{e}}, \mathbf{n}_{S}\right) R_{e}^{i} f_{e} . R_{e}^{i}$ is the right invariant vector field acting on the copy of $S U(2)$ associated to $e$. Moreover $\varepsilon\left(\dot{\mathbf{e}}, \mathbf{n}_{S}\right)=\operatorname{sgn}\left(n_{S}^{a} \dot{e}_{a}\right)$ denotes the relative orientation of surface normal $\mathbf{n}_{S}$ and edge tangent $\dot{\mathbf{e}}$.

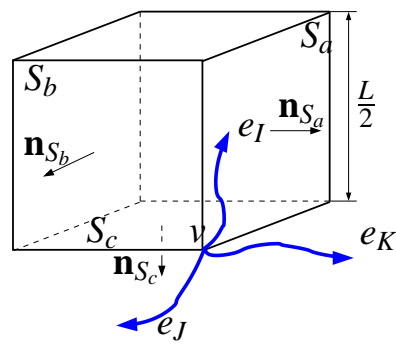

Figure 2: Regularization scheme of the classical volume expression by a cubic cell decomposition of $\Sigma_{t}$ due to [5]: The center of a cube $c_{I}^{(L)}$ contains a vertex $\mathrm{v}$. The figure only shows one octant of the cube $c_{I}^{(L)}$.

This results in an action

$$
\left(\int_{c_{I}^{(L)}} \widehat{\sqrt{|q(x)|}}\right) T_{\gamma}(A)=\sum_{e_{I} \cap e_{j} \cap e_{K}=v} \sqrt{|Z \cdot \widehat{q}|}(v) T_{\gamma}(A)
$$

where $Z$ is a constant given by $Z=$ i $C_{\text {reg }} \ell_{P}^{6} \beta^{3}$. Here the Planck length $\ell_{P}$, the Immirzi parameter $\beta \in \mathbb{R}$ and $C_{\text {reg }}$, a regularization constant (from averaging over all cube orientations) occur. The latter was found in [9] to be $\frac{1}{48}$. In the sequel we will set $Z=1$ for simplicity. Moreover

$$
\begin{aligned}
\widehat{q}(v) T_{\gamma}(A) & :=\left(\varepsilon_{a b c} \varepsilon_{i j k} \varepsilon\left(\dot{e}_{I}, \mathbf{n}_{S_{a}}\right) \varepsilon\left(\dot{e}_{J}, \mathbf{n}_{S_{b}}\right) \varepsilon\left(\dot{e}_{K}, \mathbf{n}_{S_{c}}\right) R_{e_{I}}^{i} R_{e_{J}}^{j} R_{e_{K}}^{k}\right) T_{\gamma}(A) \\
& =:\left(\varepsilon_{i j k} \varepsilon(I J K) R_{e_{I}}^{i} R_{e_{J}}^{j} R_{e_{K}}^{k}\right) T_{\gamma}(A) \\
& =:\left(\varepsilon(I J K) \widehat{q}_{I J K}\right) T_{\gamma}(A) .
\end{aligned}
$$

Here $\varepsilon(I J K)=\operatorname{sgn}\left(\operatorname{det}\left(\dot{\mathbf{e}}_{I}, \dot{\mathbf{e}}_{J}, \dot{\mathbf{e}}_{K}\right)\right)$, denotes the relative orientation of the edge tangents at $v$. By the Peter-Weyl theorem, every $T_{\gamma}(A)$ can be written in terms of products of representation matrix element functions $\left[\pi_{j}\left(h_{e}\right)\right]_{m n}$ (here $j=0, \frac{1}{2}, 1, \ldots$ denotes the weight of the $2 j+1$ dimensional irreducible representation $\pi(\cdot)$ of $S U(2), h_{e}=h_{e}(A) \in S U(2)_{e}$ is the parallel transport of the Ashtekar connection $A_{a}^{i}$ along an edge $e \subset \Sigma, m, n=-j,-j+1, \ldots, j-1, j$ are matrix indices). The right invariant vector fields $R_{e}^{i}$ only act non-trivially on the copy $S U(2)_{e}$, labelled by the edge $e$. Consequently Leibnitz' rule for derivatives gives the total local volume action as a sum over all possible triples. The fact that inside a cube $c_{I}^{(L)}$ the action of $\widehat{q}_{I J K}$ is non trivial at the vertex $v$ only follows from its antisymmetry: It only acts as a derivation non-trivially at a point, if all edge labels are 
mutually different. This condition is only fulfilled in a point, where at least 3 edges intersect, that is a vertex. Hence in the regularization procedure above the limit $L \rightarrow 0$ can be taken without problems, because in the sum over all cubes only a finite number of terms gives a non zero contribution. Moreover the choice of cell decomposition and coordinates is irrelevant for the result. Finally we can write the total action of the volume operator on a spin network function as

$$
\widehat{V}_{R} T_{\gamma}(A)=\left[\sum_{\nu \in V(\gamma)} \sqrt{\left|\sum_{e_{I} \cap e_{J} \cap e_{K}=v} \varepsilon(I J K) \widehat{q}_{I J K}\right|}\right] T_{\gamma}(A) .
$$

As can be shown [6], $\widehat{V}_{R}$ is compatible to the projective structure of the kinematic Hilbert space $\mathscr{H}_{0}$, that is $\widehat{V}_{R}$ is cylindrically consistent. The square root in (2.4) has now to be understood in terms of operators: one has to find an eigenbasis in which the operator $\left|\sum_{e_{I} \cap e_{J} \cap e_{K}=\nu} \varepsilon(I J K) \widehat{q}_{I J K}\right|$ is diagonal and then to take the square root in the sense of a matrix function. We see, that the action of $\widehat{V}_{R}$ decays into local actions at single vertices. Hence in order to analyze its action on a cylindrical function $T_{\gamma}(A)$ supported on an arbitrary embedded graph $\gamma$ it is sufficient to restrict our analysis to single vertices.

Alternative regularization. By a slightly different procedure [4] one obtains an expression $\widehat{q}(v) T_{\gamma} \propto \sum_{e_{I} \cap e_{J} \cap e_{K}=v} \sqrt{\left|\widehat{q}_{I J K}\right|}$. The two operators are structurally similar, but their properties differ for (gauge invariant) vertices with more than (4) 3 edges. There is no dependence on the signs $\varepsilon(I J K)$ : the volume in the regularization of [4] scales with the valence. This may lead to difficulties if one wants to consider vertices with infinitely many edges. The absence of sign factors was also found to lead to inconsistencies, if one wants the volume operator to be consistent with the flux operator in the sense of [9]: Regarding the volume as fundamental, one can use the the classical expressions $e_{b}^{j} \propto\left\{A_{a}^{i}, V_{R}\right\}, E_{i}^{a}=\sqrt{\operatorname{det} q} e_{i}^{a}=\frac{1}{2} \varepsilon_{i j k} \varepsilon^{a b c} e_{b}^{j} e_{c}^{k}$, in order to define an alternative flux operator. It was found in [9], that only the sign dependent version of $\widehat{V}_{R}$ leads to a consistent (in the sense that the flux operators coincide) construction. Also in the implementation of the Hamilton constraint as an operator the sign dependence seems to be crucial [1], in order to cancel contributions from planar 3-valent vertices in the regularization procedure [1].

For these reasons we will consider the sign dependent version of the volume due to Ashtekar and Lewandowski [5] in this lecture.

\section{Evaluation of Matrix Elements}

In order to perform the spectral analysis of $\widehat{V}_{R}$ in (2.4) one has to find an explicit formula for the matrix elements of the operators $\widehat{q}_{I J K}$ as well as to handle the sign factors $\varepsilon(I J K)$ in full generality.

\subsection{Matrix Elements for $\widehat{q}_{I J K}$ : Representation Theory}

In order to analyze $\widehat{q}_{I J K}$ one can use the correspondence matrix element functions $\left[\pi_{j}\left(h_{e}\right)\right]_{m n}$ of irreducible representations of $S U(2)$ to states $|j, m ; n\rangle$ of an abstract angular momentum system [6]. We have $\sqrt{2 j+1}\left[\pi_{j}\left(h_{e}\right)\right]_{m n} \equiv\left\langle h_{e} \mid j, m ; n\right\rangle_{e}$ and the action of right invariant vectorfields $R_{e_{I}}^{i}$ on spin network functions then corresponds to the action of angular momentum operators $J_{e_{I}}^{i}$ on the angular momentum states well known from quantum mechanics. In this correspondence, a spin 
network function at a given $N_{v}$-valent vertex $v$ can be written as $T_{v \vec{j} \vec{m} \vec{n}}(A)=\bigotimes_{k=1}^{N_{v}}\left\langle A \mid j_{k}, m_{k} ; n_{k}\right\rangle$, which is also called the tensor basis of the kinematic Hilbert space $\mathscr{H}_{0}$ at $v$. As is well known, the tensor product of two $S U(2)$ irreducible representations of weight $j_{1}, j_{2}$ can be decomposed into a direct sum of irreducible representations with weights $a_{2}=\left|j_{1}-j_{2}\right|, \ldots, j_{1}+j_{2}$. In this way a tensor basis function containing $N_{v}$ irreducible representations can be successively decomposed as shown in figure 3.

\begin{tabular}{cc}
\multicolumn{2}{c}{$j_{1}$} \\
\hline$j_{2}$ \\
\hline$j_{3}$ \\
\hline$j_{4}$ \\
\hline$\vdots$ & \\
\hline$j_{N_{v}}$ & $\vdots$ \\
\hline
\end{tabular}

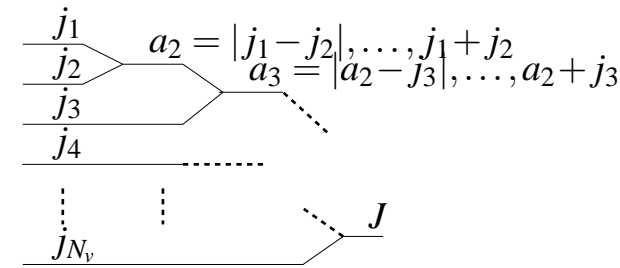

Figure 3: Tensor basis (left) and recoupling basis (right) as equivalent description.

The result is called a recoupling scheme $T_{v \vec{a} J M \overrightarrow{j \vec{n}}}(A)=\left\langle A \mid \vec{a}_{12} J M ; \vec{j} \vec{n}\right\rangle$ at the vertex $v$ and can be explicitly computed using Clebsch Gordan coefficients from the recoupling theory of angular momentum. Here $\vec{a}_{12}=\left(a_{2}, a_{3}, \ldots, a_{N_{v}-1}\right)$ is a vector of intermediate recoupling weights, $J$ is the total angular momentum and the subscript 12 denotes the fact that $j_{1}, j_{2}$ are coupled first. Moreover $M=\sum_{k=1}^{N_{v}} m_{k}$ and $\vec{n}$ is as before and used in order to connect $T_{v \vec{j} \vec{m} \vec{n}}(A)$ at different vertices of the graph $\gamma$. In this formalism the gauge invariant subspace $\mathscr{H}_{\text {Gauss }} \subset \mathscr{H}_{0}$ can be easily computed: by construction the recoupling schemes with $J=0$ fulfill the Gauss constraints $\mathscr{G}_{i}(v)$. Notice that the labelling of edges is arbitrary. Indeed, different recoupling orders, that is different labellings of edges, are connected by unitary transformations due to unitarity of the Clebsch Gordan coefficients. In the recoupling basis, the operator $\widehat{q}(V)$ of (2.3) can be explicitly evaluated using the fact that $\widehat{q}_{I J K}=\varepsilon_{i j k} J_{e_{I}}^{i} J_{e_{J}}^{j} J_{e_{K}}^{k}=\frac{\dot{i}}{4}\left[\left(J_{I J}\right)^{2},\left(J_{J K}\right)^{2}\right]$, where $J_{I J}^{i}:=J_{I}^{i}+J_{J}^{i}$ and $\left(J_{I J}\right)^{2}\left|\vec{a}_{I J} J M ; \vec{n}\right\rangle=a_{I J}\left(a_{I J}+\right.$ 1) $\left|\vec{a}_{I J} J M ; \vec{n}\right\rangle$ is diagonal on a recoupling scheme, in which the representations $j_{I}, j_{J}$ are coupled first [8]. As a consequence, the matrix elements of (2.3) can be explicitly evaluated. For a fixed choice of recoupling scheme basis for the gauge invariant Hilbert space $\mathscr{H}_{\text {Gauss }}$ the operator $\widehat{q}(v)$ can be represented as a sum of Hermitian matrices with varying pre-factors ${ }^{1}$.

$$
\widehat{q}(v) \propto\left|\sum_{I, J, K<N_{v}} \sigma(I J K) \widehat{q}_{I J K}\right|,
$$

where $\sigma(I J K)=\varepsilon(I J K)-\varepsilon\left(J K N_{v}\right)+\varepsilon\left(I K N_{v}\right)-\varepsilon\left(I J N_{v}\right)$, with $-4 \leq \sigma(I J K) \leq 4$. For the 4-vertex a formula for the matrix elements was published in [7]. A general formula for the elements of the matrices $\widehat{q}_{I J K}$ can be found in [8].

Gauge invariant 4 vertex. Equation (3.1) already suffices to analyze the volume spectrum at 4valent gauge invariant vertices: For $N_{v}=4$ only a single matrix $\widehat{q}_{123}$ needs to be computed, the sign factor $\sigma(123)$ only gives an overall scaling of the spectrum. Beside a numerical analysis $[8,14]$ one can analytically compute the subspace $\operatorname{kern}(\widehat{V}) \subset \mathscr{H}_{\text {Gauss }}$ for the eigenvalue 0 . Moreover one finds that for a given vertex $v$ the spectrum of $\widehat{V}$ is non degenerate. The spectrum possesses a smallest non zero eigenvalue $\lambda_{\min } \geq \ell_{P}^{3} \sqrt{|Z \sigma(123)| j_{\max }}$, where $j_{\max }=\max _{K=1 \ldots N_{v}}\left\{j_{K}\right\}$. For the largest eigenvalue one finds $\lambda_{\max } \propto\left(j_{\max }\right)^{3 / 2}$.

\footnotetext{
${ }^{1}$ In case of gauge invariance we have $J=0$ and hence $J_{e_{N_{v}}}^{i}=-\sum_{K=1}^{N_{v}} J_{e_{K}}$.
} 


\subsection{Arbitrary Valence: Sign Factor Combinatorics in Terms of Oriented Matroids}

For a general valence $N_{v}>4$ an explicit computation of (3.1) is much more involved. We have already seen, that edge relabellings and analytic diffeomorphisms act unitarily on $\mathscr{H}_{0}$ and thus on $\mathscr{H}_{\text {Gauss. }}$. Hence we need to classify all diffeomorphism equivalence classes of embeddings of an $N_{v^{-}}$ valent vertex $v$ into $\Sigma$. Each such class is described by a set $\vec{\sigma}$ of sign factors $\vec{\sigma}:=\left\{\sigma_{I J K}\right\}_{I, J, K<N_{v}}$, which is diffeomorphism invariant by construction. However the signs $\sigma(I J K)$ are not invariant under edge-relabelling ( $\equiv$ permutation of edge labels): each orbit of $\vec{\sigma}$ under the permutation group $\mathscr{P}_{N_{v}}$ over $N_{v}$ elements gives one presumably distinct volume spectrum. As we will show in this section, the classification of all such permutation equivalence classes of sign factors is a highly non-trivial combinatorial task. As it turns out [15], the solution corresponds to a classification of all oriented matroids [2] of rank 3 .

Linear Dependence of Vector Configurations. In order to describe the sign factor properties, we now give an abstract combinatorial description of linear dependencies among vector configurations in $\mathbb{R}^{3}$. Given a sorted ${ }^{3}$ set $E=\left(\mathbf{e}_{1}, \ldots, \mathbf{e}_{N}\right) \subset \mathbb{R}^{3}$ of $N$ vectors, we define its family $\mathscr{B}$ of oriented bases by $\mathscr{B}=\left\{B=\left(\mathbf{e}_{I}, \mathbf{e}_{J}, \mathbf{e}_{K}\right)_{I<J<K} \subseteq E: B\right.$ spans $\left.\mathbb{R}^{3}\right\}$. For every set $S \subseteq E$ a basis orientation $\chi_{\mathscr{B}}$ : $S \rightarrow\{0, \pm 1\} \quad$ is defined as $\chi_{\mathscr{B}}(S)=1$ or -1 iff $S \in \mathscr{B}$ and 0 if $S \notin \mathscr{B}$. The map $\chi_{\mathscr{B}}$ is also called chirotope in the literature. The notion of positive or negative sign of a basis orientation is subject to a choice. For $N=5$ on the left in figure 4 we have $\mathscr{B}=\{(123),(124),(134),(135),(145),(234),(235),(245)\}$, and $\chi_{\mathscr{B}}(B)= \pm \operatorname{sgn}(\operatorname{det} B)$, with $\overrightarrow{\chi_{\mathscr{B}}}= \pm(+,+,-,-,-,+,+,+)$. To fix the sign in front corresponds to our choice of positive orientation (right or left handed) of a dreibein.

Equivalently we may characterize the vector configuration in figure 4 in terms of its set $\mathscr{C}$ of signed circuits, where $\mathscr{C}:=\{C \subseteq E: C$ is minimum linear dependence $\}$ : Given a set $C \subseteq E$ with cardinality $N_{C}$, we say that $C=\left\{\mathbf{e}_{K_{1}}, \ldots, \mathbf{e}_{K_{N_{C}}}\right\}$ is a linear dependence if there exists a set $\left(\lambda_{1}, \ldots, \lambda_{N_{C}}\right) \in \mathbb{R}^{N_{C}}$, such that $0=\sum_{n=1}^{N_{C}} \lambda_{n} \mathbf{e}_{K_{n}} . C$ is a minimum linear dependence if $\lambda_{n} \neq 0 \forall n$ and if $C$ does not contain dependent subsets. Then we can write $C$ in terms of its signed subsets: $C=\left\{C^{+}, C^{-}\right\}$, where $C^{ \pm}:=\left\{\mathbf{e}_{K_{n}}: \lambda_{n} \gtrless 0\right\}$. Moreover we define the support ${ }^{4} \operatorname{supp} C=$ $\underline{C}:=C^{+} \cup C^{-}$. Now given a signed circuit $C \in \mathscr{C}$ we define for every $S \subseteq E$ a map $\operatorname{sgn}_{C}: S \rightarrow$ $\{0, \pm 1\}$ as $\operatorname{sgn}_{C}\left(\mathbf{e}_{K}\right)= \pm 1$ iff $\mathbf{e}_{K} \in C^{ \pm}$and 0 else. This classification is again defined only up to a global sign: Indeed it must hold that $(-C)^{ \pm}=C^{\mp}$. Hence if $C=\left\{C^{+}, C^{-}\right\} \in \mathscr{C}$ then also $-C:=\left\{-C^{+},-C^{-}\right\}=\left\{C^{-}, C^{+}\right\} \in \mathscr{C}$. For the example of figure 4 one finds $\mathscr{C}=\left\{ \pm C_{1}, \pm C_{2}, \pm C_{3}\right\}$ with $C_{1}=\{\{123\},\{4\}\}, C_{2}=\{\{12\},\{5\}\}$ and $C_{3}=\{\{35\},\{4\}\}$.

As it turns out, the two descriptions of the vector configuration in terms of oriented bases $\mathscr{B}$ and signed circuits $\mathscr{C}$ are equivalent: For every $B \in \mathscr{B}$ and for every $\mathbf{e} \in E \backslash B$ there is a unique $\pm C \in \mathscr{C}$ such that $B \cup\{\mathbf{e}\} \subseteq \underline{C}$. Given two bases $B_{1}, B_{2} \in \mathscr{B}$ with $B_{1}=\left(\mathbf{e}_{I}, \mathbf{e}_{M}, \mathbf{e}_{N}\right), B_{2}=\left(\mathbf{e}_{L}, \mathbf{e}_{M}, \mathbf{e}_{N}\right)$ there exists a $\pm C \in \mathscr{C}$ with $B_{1} \cup\left\{\mathbf{e}_{L}\right\}=B_{2} \cup\left\{\mathbf{e}_{I}\right\} \subseteq \underline{C}$. Then the following identity holds [2]:

$$
\operatorname{sgn}_{C}\left(\mathbf{e}_{I}\right) \operatorname{sgn}_{C}\left(\mathbf{e}_{L}\right)=\chi_{\mathscr{B}}\left(B_{1}\right) \chi_{\mathscr{B}}\left(B_{2}\right)
$$

\footnotetext{
${ }^{2}$ Which is essential in order to describe graph changing operators such as the Hamilton constraint operator.

${ }^{3}$ With respect to an arbitrarily chosen label set.

${ }^{4}$ By construction $\underline{C}=\underline{-C}$.
} 

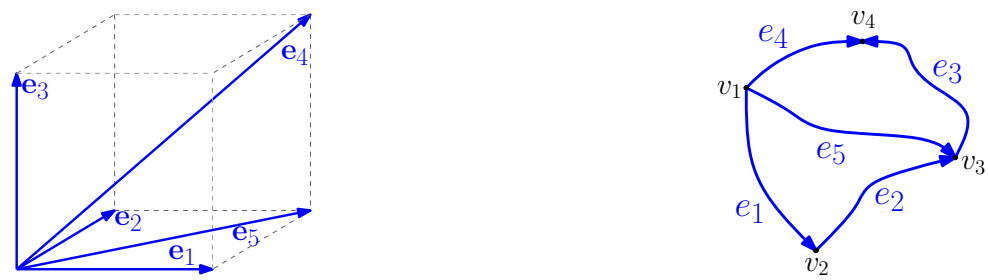

Figure 4: Oriented matroid combinatorial framework. Left: vector representation of $\mathscr{M}$. Right: graph representation of $\mathscr{M}$. Throughout the text we use the shorthand $K \equiv \mathbf{e}_{K}$.

Using (3.2) one can convert between the two descriptions of a vector configuration. Notice that the formalism described here can be easily generalized to vector configurations in $\mathbb{R}^{N}$ with arbitrary $N>0$.

Definition of an Oriented Matroid. The definition outlined so far gives rise to the definition of an oriented matroid: A family $\mathscr{C}$ of signed subsets $C$ of a finite ground set $E$ is called the set of signed circuits of an oriented matroid $\mathscr{M}=(E, \mathscr{C})$ if the circuit axioms $(C 0) \ldots(C 3)$ hold:

$$
\begin{array}{ll}
\text { (CO) Non emptiness: } & \emptyset \notin \mathscr{C} \\
\text { (C1) Symmetry: } & \mathscr{C}=-\mathscr{C} \Leftrightarrow \pm C \in \mathscr{C} \\
\text { (C2) Incomparability: } & \frac{C_{1}}{\forall}=\underline{C_{2}} \Leftrightarrow C_{1}= \pm C_{2} \quad \forall C_{1}, C_{2} \in \mathscr{C} \\
\text { (C3) Elimination: } & \forall C_{1}, C_{2} \in C \text { with } C_{1} \neq \pm C_{2}: \forall \mathbf{e} \in C_{1}^{+} \cap C_{2}^{-} \quad \exists C_{3} \in \mathscr{C}: \\
& C_{3}^{ \pm} \subseteq\left\{C_{1}^{ \pm} \cup C_{2}^{ \pm}\right\} \backslash\{\mathbf{e}\}
\end{array}
$$

A similar definition $\mathscr{M}=(E, \mathscr{B})$ of $\mathscr{M}$ can be given in terms of its oriented bases $\mathscr{B}[2,15]$. Notice that the definition of an oriented matroid is purely combinatorial. In particular it does not refer to a vector configuration any more. Indeed, a vector configuration can be understood as a realization of an abstract combinatorial oriented matroid $\mathscr{M}$ in terms of vectors [2]. However as it turns out there there are far more realizations, all of which can be commonly described by oriented

\begin{tabular}{|c|c|c|c|c|c|}
\hline$N_{v}$ & \# triples & $\# \vec{\varepsilon}\left(N_{v}\right)$ sprinkled & $\begin{array}{l}\# \vec{\varepsilon} \text { perm. } \\
\text { equiv. classes }\end{array}$ & $\# \vec{\sigma}$ configs & $\begin{array}{l}\text { \# realizable reor. } \\
\text { equiv. classes }\end{array}$ \\
\hline 3 & 1 & 2 & 1 & 1 & 1 \\
\hline 4 & 4 & 16 & 3 & 3 & 1 \\
\hline 5 & 10 & 384 & 4 & 4 & 1 \\
\hline 6 & 20 & 23808 & 41 & 39 & 4 \\
\hline 7 & 35 & 3486720 & 706 & 673 & 11 \\
\hline
\end{tabular}
matroids. This aspect will be further discussed in section 5 .

Results for $\hat{V}$. Using the oriented matroid framework one can compare numerical results [15] on possible sign factor configurations $\vec{\sigma}$ to results in the oriented matroids literature $[16,17]$. A summary is given in table 1 . Column 3 shows the number of possible $\vec{\varepsilon}:=\left\{\varepsilon_{I J K}\right\}_{I, J, K \leq N_{v}}$ con-

Table 1: Sign factor combinatorics for 3-7-valent non-coplanar vertices, corresponding to the classification of uniform rank 3 oriented matroids.

figurations obtained from sprinkling points on the unit sphere using Monte Carlo methods [14]. Column 4 shows the number of equivalence classes if the $N_{v}$-element permutation group $\mathscr{P}_{N_{v}}$ is 
factored out. Column 5 shows the corresponding number of $\vec{\sigma}:=\left\{\sigma_{I J K}\right\}_{I, J, K<N_{v}}$ - configuration for a gauge invariant $N_{v}$-valent vertex. Column 6 gives results from [16]: there the reorientation of single elements is additionally factored out from the permutation equivalence classes. For a vector configuration, this can be understood as the flipping of one vector at the origin to its negative direction. By factoring out this additional symmetry, one obtains column 6 from column 4. However, for the case of a $N_{v}$-valent vertex $v$ reorientation does not mean reorientation of an edge. Rather it means [15] that the tangent of an edge $e$ adjacent to $v$ is replaced by the tangent of a different edge $e^{\prime}$. Hence the spectrum of $\hat{q}_{v}$ of equation (3.1) is not invariant under reorientations. It is only invariant under label permutations. Therefore the number of distinct spectra of $\hat{q}_{v}$ at a given vertex $v$ is given by the number of permutation equivalence classes of the sign factors $\vec{\sigma}$.

\section{Spectral Properties}

Having both at hand, matrix elements from recoupling theory and sign factors from oriented matroid theory it is possible to numerically compute the volume spectrum for all non diffeomorphically embedded vertices $v$ with arbitrary valence $N_{v}$. As an example, results from [14] for $N_{v}=5$ are given in figures 5 and 6 . Notice that we only have 3 non-trivial spectra (labelled by the $\vec{\sigma}$-index $0,1,2$ ), because one $\vec{\sigma}$ sign configuration at a vertex (the one where all tangent vectors have the same relative orientation) is always trivial.

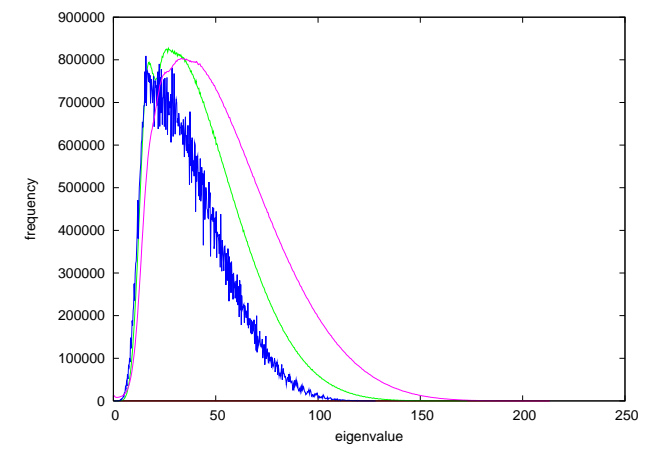

Figure 5: Histograms for each sigma configuration $\vec{\sigma}$ at the generic (gauge invariant) 5-vertex up to $j_{\max }=25 / 2$.

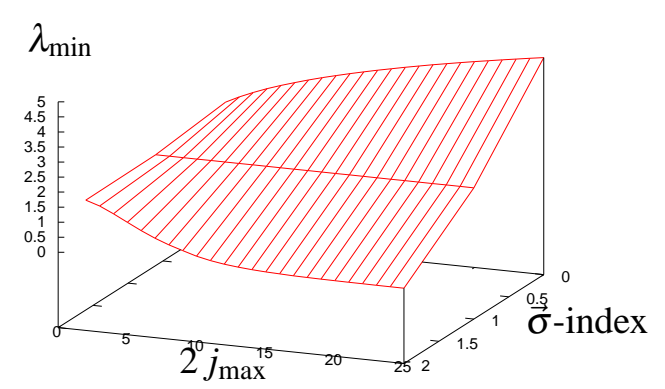

Figure 6: Smallest non-zero eigenvalues $\lambda_{\min }$ at the (gauge invariant) 5-vertex

Figure 5 shows the volume spectrum for the generic gauge invariant 5 -vertex. Here 'generic' means excluding co-planar edges. We use the notation $\vec{\sigma}:=\left(\sigma_{123}, \sigma_{124}, \sigma_{134}, \sigma_{234}\right)$. The green color is for $\vec{\sigma}_{0}=(2,2,2,0)$, the blue is for $\vec{\sigma}_{1}=(2,0,0,0)$ and the purple for $\vec{\sigma}_{2}=(2,2,4,0)$. Each histogram has 512 bins. As it is obvious from the figures, the permutation equivalence class of the sign factors $\vec{\sigma}$ determines the overall shape of the spectrum at a given vertex. In particular the presence of a smallest non-zero eigenvalue $\lambda_{\min }$ (volume gap) depends on the sign factors. This can be already seen for $N_{v}=5$ in figure 6 , where we find $\lambda_{\min }$ increasing, decreasing or constant as the maximum spin $j_{\max }$ at the vertex is increased. 


\section{Conclusions \& Outlook}

Although technically involved, the spectrum of the volume operator of LQG is accessible for a complete numerical study. As it turns out, embedding properties of vertices determine the spectral properties, in particular the presence of a volume gap depends of the permutation equivalence class of signs. Taking the volume spectrum as fundamental this implies that the volume spectrum contains (spatially diffeomorphism invariant) geometric information of the tangent space at a vertex $v$. First steps towards a numerical study of the volume spectrum have already been performed in $[14,15]$ for non co-planar (all triples of edge tangent linearly independent $\equiv$ uniform underlying oriented matroid) vertices. However in the oriented matroid literature $[16,17]$ sign data is also available for non uniform oriented matroids, that is vertices with several co-planar triples of edge tangents. Hence the spectral analysis of the volume operator needs to be extended to these cases. Having this at hand semiclassical results for the volume spectrum [11, 18], which had been restricted to only a few vertex embeddings, can now be checked in full generality.

As we have mentioned above, the abstract concept of an oriented matroid $\mathscr{M}$ has distinct realizations apart from a vector configuration. From the LQG view point the most interesting case is the realization of $\mathscr{M}$ in terms of a directed graph $\gamma$ instead of a vector configuration in $\mathbb{R}^{N}$. The ground set $E$ of $\mathscr{M}$ is then given by the set $E(\gamma)$ of edges, the set $\mathscr{C}$ of signed circuits (min. linear dependencies) is identified with the set of cycles in the graph. The set $\mathscr{B}$ of oriented bases (spanning subsets) is identified with the set of spanning trees of $\gamma$. The dimension $N$ of $\mathbb{R}^{N}$ in the vector representation gives the cardinality of elements $B \in \mathscr{B}$, called the rank of $\mathscr{M}$. In the case of a directed graph $N+1$ is the number of vertices of the corresponding directed graph. As an example, the vector realization of an oriented matroid of rank 3 as well as its corresponding realization as a directed graph with 4 vertices are given in figure 4 .

As it has been explained in the introductory lectures for LQG, the construction of the kinematical Hilbert space $\mathscr{H}_{0}$ rests on the projective limit among a label set consisting of embedded directed graphs. Here a completely new perspective towards a rigorous combinatorial formulation of LQG is opened by the oriented matroid framework, if we can describe this projective limit in terms of a projective limit among oriented matroids. Generalizations of the matroid framework to infinite ground (edge-) sets have already been investigated in the mathematics literature [19].

This analysis has the potential to shed light on the question, if the duality between graphs (topology) and vertices (local embedding) has deeper implications for quantum gravity. This will be subject to future research.

\section{Acknowledgments}

The author is grateful to the organizers of this stimulating school for giving him the opportunity to present his work in a lecture. Furthermore the author is grateful to D. Rideout for a long term fruitful collaboration. This work has been supported by by the Emmy-Noether-Programm (grant FL 622/1-1) of the Deutsche Forschungsgemeinschaft. The numerical results were generated using the facilities of the Shared Hierarchical Academic Research Computing Network (SHARCNET:www.sharcnet.ca). The author also thanks the team of XRG for support of this work and the anonymous referee for helpful comments. 


\section{References}

[1] T. Thiemann. Modern Canonical Quantum General Relativity. Cambridge University Press, 2007.

[2] A. Björner, M. Las Vergnas, B. Sturmfels, N. White, and G. Ziegler. Oriented Matroids. Cambridge University Press, second edition, 1999.2

[3] A. Ashtekar and J. Lewandowski, "Differential Geometry on the Space of Connections via Graphs and Projective Limits", J. Geom. Phys. 17 (1995) 191-230; [arXiv: hep-th/9412073v2 ].

[4] C. Rovelli and L. Smolin, "Discreteness of area and volume in quantum gravity", Nucl. Phys. B 442 (1995) 593 [Erratum-ibid. B 456 (1995) 753]; [arXiv : gr-qc/9411005] .

[5] A. Ashtekar and J. Lewandowski, "Quantum theory of geometry. II: Volume operators", Adv. Theor. Math. Phys. 1, 388 (1998); [arXiv:gr-qc/9711031].

[6] T. Thiemann, "Closed formula for the matrix elements of the volume operator in canonical quantum gravity”, J. Math. Phys. 39, 3347 (1998); [arXiv: gr-qc/9606091] .

[7] R. De Pietri, "Spin Networks and Recoupling in Loop Quantum Gravity”, Nucl. Phys. Proc. Suppl. 57, 251 (1997); [arXiv:gr-qc/9701041].

[8] J. Brunnemann and T. Thiemann, "Simplification of the spectral analysis of the volume operator in loop quantum gravity”, Class. Quant. Grav. 23 (2006) 1289; [arXiv:gr-qc/ 0405060 ] .

[9] K. Giesel and T. Thiemann, "Consistency check on volume and triad operator quantisation in loop quantum gravity. I”, Class. Quant. Grav. 23 (2006) 5667; [arXiv:gr-qc/0 507036 ] .

[10] K. Giesel, S. Hofmann, T. Thiemann and O. Winkler, "Manifestly Gauge-Invariant General Relativistic Perturbation Theory. I. Foundations,” Class. Quant. Grav. 27 (2010) 055005; [arXiv:0711.0115 [gr-qc]].

[11] K. A. Meissner, "Eigenvalues of the volume operator in loop quantum gravity", Class. Quant. Grav. 23 (2006) 617; [arXiv:gr-qc/0509049].

[12] Tim A. Koslowski, "Dynamical Quantum Geometry (DQG Programme)", [arXiv:0709.3465v1 [gr-qc]]

[13] Eugenio Bianchi, Hal M. Haggard, "Discreteness of the volume of space from Bohr-Sommerfeld quantization”, Phys.Rev.Lett.107:011301,2011, [arXiv:1102.5439v1 [gr-qc] ],

[14] J. Brunnemann and D. Rideout, "Properties of the Volume Operator in Loop Quantum Gravity I: Results", Class. Quant. Grav.25:065001,2008, [ [arXiv:0706.0469v1] [gr-qc] ] .

[15] J. Brunnemann and D. Rideout, “Oriented Matroids - Combinatorial Structures Underlying Loop Quantum Gravity”, Class.Quant.Grav.27:205008,2010 [arXiv:1003.2348v2 [gr-qc] ]

[16] L. Finschi. "A Graph Theoretical Approach for Reconstruction and Generation of Oriented Matroids." PHD thesis, Swiss Federal Institute of Technology (ETH), Zurich, 2001.

[17] L. Finschi and K. Fukuda. "Generation of Oriented Matroids - A Graph Theoretical Approach." Discrete Comput Geom, 27: 117 - 136, 2002.

[18] C. Flori, T. Thiemann, "Semiclassical analysis of the Loop Quantum Gravity volume operator: I. Flux Coherent States", [arXiv:0812.1537v1 [gr-qc] ] .

[19] H. Bruhn, R. Diestel, M. Kriesell, R. Pendavingh and P. Wollan. "Axioms for infinite matroids." [arXiv:1003.3919v2[math.CO]]. 